\title{
Theory of planet formation and comparison with observation
}

\author{
C. Mordasini ${ }^{1}$, Y. Alibert ${ }^{2}$, H. Klahr ${ }^{1}$, W. Benz ${ }^{2}$ \\ ${ }^{1}$ Max Planck Institute for Astronomy, Königstuhl 17, D-69117 Heidelberg, \\ Germany [mordasini@mpia.de]
}

\section{Physikalisches Institut, Sidlerstrasse 5, CH-3012 Bern, Switzerland}

\begin{abstract}
The planetary mass-radius diagram is an observational result of central importance to understand planet formation. We present an updated version of our planet formation model based on the core accretion paradigm which allows us to calculate planetary radii and luminosities during the entire formation and evolution of the planets. We first study with it the formation of Jupiter, and compare with previous works. Then we conduct planetary population synthesis calculations to obtain a synthetic mass-radius diagram which we compare with the observed one. Except for bloated Hot Jupiters which can be explained only with additional mechanisms related to their proximity to the star, we find a good agreement of the general shape of the observed and the synthetic $M-R$ diagram. This shape can be understood with basic concepts of the core accretion model.
\end{abstract}

\section{Introduction}

The number of known transiting extrasolar planets is increasing rapidly. Combined with radial velocity measurements which yield the mass of the planet, one gets the planetary mass-radius diagram, which is an observational result of similar importance as the semimajor axis-mass diagram. The reason for this is that one can derive the mean density of the planet, which constrains, at least to some extent, the internal structure which is of central importance to understand the nature (Leconte et al. 2009) and, as we shall see, the formation of the planet. In this work we discuss recent steps towards a synthetic mass-radius diagram as obtained through planetary population synthesis. Keeping in mind the goal of the colloquium, we concentrate the comparison of theoretical and observed results on this subject. A comparison of numerous other theoretical and observational results mostly obtained by the radial-velocity technique can be found in Mordasini et al. (2009b).

Before addressing results obtained by the population synthesis, it is essential to present the basic concepts of the planet formation theory, and how they are described in the formation model. As most transiting extrasolar planets are still giant planets, it is natural to focus on the formation of this type of planets. 


\section{EPJ Web of Conferences}

\section{Giant planet formation model}

The results presented here were obtained using an updated version of the planet formation code of Alibert, Mordasini, \& Benz (2004), described in details in Alibert et al. (2005). In this version (Mordasini et al. in prep.), we have added the calculation of the planetary structure during the full formation and subsequent evolution of the planet. This allows to calculate planetary radii (instead of the mass only as in previous versions) which is obviously of primary interest for transit observations.

The formation model used here relies on the core accretion paradigm. Its basic conception is to follow the concurrent growth of an initially small solid core consisting of ices and rocks, and its surrounding gaseous envelope, embedded in the protoplanetary disk (Perri \& Cameron 1974; Mizuno et al. 1978; Bodenheimer \& Pollack 1986, hereafter $\mathrm{BP} 86)$.

\subsection{Core growth}

The growth rate of the solid core is described with a Safronov (1969) type rate equation,

$$
\frac{d M_{\mathrm{Z}}}{d t}=\pi R_{\mathrm{capt}}^{2} \Omega \Sigma_{\mathrm{p}} F_{\mathrm{G}}
$$

(cf. Lissauer 1993) where $\Omega$ is the Keplerian frequency of the protoplanet at an orbital distance $a$ around the star of mass $M_{*}, \Omega=\sqrt{G M_{*} / a^{3}}, \Sigma_{p}$ is the surface density of the field planetesimals, $R_{\text {capt }}$ is the capture radius of the embryo, and $F_{\mathrm{G}}$ is the gravitational focussing factor (Greenzweig \& Lissauer 1992).

In previous versions of the formation model, we assumed a constant core density of $3.2 \mathrm{~g} / \mathrm{cm}^{3}$. In order to obtain radii also for solid dominated lower mass planets, we have replace this assumption with an internal structure model for the core. This model was originally developed for the study of GJ 436b in Figueira et al. (2009). We include the effect of the external pressure by the envelope, which leads to significant compression of the core for giant planets (Baraffe, Chabrier, \& Barman 2008).

\subsection{Envelope growth}

The gas accretion rate of the planet (at least in the early phases, cf. below) is obtained by solving the one dimensional, hydrostatic planetary structure equations. These equations are similar to those for the interiors of stars, except that the energy release by nuclear fusion is replaced by the heating by impacting planetesimals $\epsilon$, which are an important energy source during the early formation stage. The other equations are the standard equations of mass conservation, of hydrostatic equilibrium, of energy conservation and of energy transfer which are given as (e.g. BP86; Guillot 2005; Broeg 2009):

$$
\begin{array}{ll}
\frac{d m}{d r}=4 \pi r^{2} \rho & \frac{d P}{d r}=-\frac{G m}{r^{2}} \rho \\
\frac{d l}{d r}=4 \pi r^{2} \rho\left(\epsilon-T \frac{\partial S}{\partial t}\right) & \frac{d T}{d r}=\frac{T}{P} \frac{d P}{d r} \nabla
\end{array}
$$

In these equations, $r$ is the radius as measured from the planetary center, $m$ the mass inside $\mathrm{r}$ (including the core mass $M_{\mathrm{Z}}$ ), $l$ the luminosity at $r$, and $\rho, P, T, S$ are the gas density, pressure, temperature and entropy, $t$ the time, and $\nabla$ is given as

$$
\nabla=\frac{d \ln T}{d \ln P}=\min \left(\nabla_{\mathrm{ad}}, \nabla_{\mathrm{rad}}\right) \quad \nabla_{\mathrm{rad}}=\frac{3}{64 \pi \sigma G} \frac{\kappa l P}{T^{4} m}
$$

i.e. by the minimum of the adiabatic gradient $\nabla_{\text {ad }}$ which is directly given by the equation of state (in convective zones) or the radiative gradient $\nabla_{\text {rad }}$ (in radiative zones) where $\kappa$ is the opacity and $\sigma$ is the Stefan-Boltzmann constant. 


\section{Calculation of the luminosity}

For the planetary population synthesis, where the evolution of thousands of different planets is calculated, we need a stable and rapid method for the numerical solution of these equations. We have therefore replaced the ordinary equation for $d l / d r$ by the assumption that $l$ is constant within the envelope, and that we can derive the total luminosity $L$ (including solid and gas accretion, contraction and release of internal heat) and its temporal evolution by total energy conservation arguments, an approach somewhat similar to Papaloizou \& Nelson (2005). We first recall that $-d E_{\text {tot }} / d t=L$ and that in the hydrostatic case, the total energy is given as

$$
E_{\text {tot }}=E_{\text {grav }}+E_{\text {int }}=-\int_{0}^{M} \frac{G m}{r} d m+\int_{0}^{M} u d m \doteq-\xi \frac{G M^{2}}{2 R}
$$

where $u$ is the specific internal energy, $M$ the total mass, and $R$ the total radius of the planet. We have defined a parameter $\xi$, which represents the distribution of mass within the planet and its internal energy content. The $\xi$ can be found for any given structure at time $t$ with the equations above. Then one can write

$$
-\frac{d}{d t} E_{\mathrm{tot}}=L=L_{M}+L_{R}+L_{\xi}=\frac{\xi G M}{R} \dot{M}-\frac{\xi G M^{2}}{2 R^{2}} \dot{R}+\frac{G M^{2}}{2 R} \dot{\xi}
$$

where $\dot{M}=\dot{M}_{\mathrm{Z}}+\dot{M}_{\mathrm{XY}}$ is the total accretion rate of solids and gas, and $\dot{R}$ is the rate of change of the total radius. All quantities except $\dot{\xi}$ can readily be calculated at time $t$. We now set

$$
L \simeq C\left(L_{M}+L_{R}\right)
$$

The factor $C$ corrects that we neglect the $L_{\xi}$ term, and is obtained in this way: a posteriori, one can calculate the total energy in the new structure at $t+d t$, which gives the exact luminosity as $L_{\mathrm{ex}}=-\left[E_{\mathrm{tot}}(t+d t)-E_{\mathrm{tot}}(t)\right] / d t$. By setting $C=L_{\mathrm{ex}} / L$ one obtains the correction factor so that exact energy conservation would have occurred. As an approximation, we then use this $C$ for next time step. One finds that in this way, the estimated luminosity $L$ and the actual luminosity $L_{\text {ex }}$ agree generally very well, provided that $d t$ is small enough. Note that the release of energy by the contraction of the core - the dominant contribution from it for giant planets (Baraffe et al. 2008) - is automatically included in the total luminosity in this method.

We have tested the impact of the simplified luminosity calculation by comparison with calculations based on the full set of structure equations and found good agreement (Broeg et al. in prep, sect. 3.). The assumption that $d l / d r=0$ has a small effect because of the following: The radial variation of the luminosity is only relevant in the radiative zones of the planet (see eq. 3). Significant radiative zones however only exist during the early phase of the planet's formation (BP86). But then, a large part of the luminosity is generated by the core luminosity, so our approximation is close to the exact solution. During the long term evolution, the planet is nearly fully convective (e.g. Guillot 2005), so that the radial variation of $l$ is irrelevant, too.

Finally one must take into account that a part of the released gravitational energy of newly accreted material is not incorporated into the planetary structure but already lost as $L_{\text {ext }}$ in the accretion shock on the planet's surface or in the surrounding circumplanetary disk (Bodenheimer, Hubickyj, \& Lissauer 2000; Papaloizou \& Nelson 2005). The luminosity within the planetary structure is then $L_{\text {int }}$ where

$$
L_{\mathrm{ext}}=\frac{G M}{R} \dot{M}_{\mathrm{XY}} \quad L_{\mathrm{int}}=L-L_{\mathrm{ext}}
$$


while an observer would see the total luminosity $L$. It is clear that this treatment is a strong simplification of the exact physics of the accretion shock (e.g. Stahler, Shu, \& Taam 1980).

As one can see, we do not include any mechanism which could lead to the so called "radius anomaly" observed for many transiting Hot Jupiter which have radii clearly larger than expected from standard internal structure modeling as done here. The physical mechanisms leading to this bloating are not yet understood, and discussed in dedicated studies (e.g. Leconte et al., this volume).

\subsection{Boundary conditions}

For the formation and evolution of a giant planet, three different fundamental phases must be distinguished:

\section{Attached (or nebular) phase}

At low masses, the envelope of the protoplanet is attached continuously to the background nebula, and the conditions at the surface of the planet are the pressure $P_{\text {neb }}$ and approximately the temperature $T_{\text {neb }}$ in the surrounding disk. The total radius $R$ is given in this regime by roughly the minimum of the Hill radius $R_{\mathrm{H}}$ and the accretion radius $R_{\mathrm{A}}$. The gas accretion rate is found by the solution of the structure equations and physically given by the ability of the envelope to radiate away energy so that it can contract (i.e. its Kelvin-Helmholtz timescale), so that new gas can stream in (Pollack et al. 1996). In equations, we use (Bodenheimer et al. 2000; Papaloizou \& Terquem 1999)

$$
\begin{array}{ll}
R=\frac{R_{\mathrm{A}}}{1+R_{\mathrm{A}} / R_{\mathrm{H}}} & P=P_{\text {neb }} \\
\tau=\max \left(\rho_{\text {neb }} \kappa_{\text {neb }} R, 2 / 3\right) & T_{\text {int }}^{4}=\frac{3 \tau L_{\mathrm{int}}}{8 \pi \sigma R^{2}} \\
T^{4}=T_{\text {neb }}^{4}+T_{\text {int }}^{4} & l(R)=L_{\text {int }} .
\end{array}
$$

\section{Detached (or transition) phase}

When the gas accretion obtained in this way becomes too high in comparison with the externally possible gas supply, the planet enters the second phase and contracts to a radius which is much smaller than the Hill sphere radius. This is the detached regime of high mass, runaway gas accretion planets. The planet now adjusts its radius to the boundary conditions that are given by an accretion shock on the surface for matter falling with free fall velocity $v_{\text {ff }}$ onto the planet from the Hill sphere radius, or probably more realistically, by conditions appropriate for the interface to a circumplanetary disk (Papaloizou \& Nelson 2005).

In this phase, the gas accretion rate $\dot{M}_{\mathrm{XY}}$ is no more controlled by the planetary structure itself, but by how much gas is supplied by the disk and can bypass the gap formed by the planet in the protoplanetary disk, $\dot{M}_{\text {disk }}$ (Lubow, Seibert, \& Artymowicz 1999). The boundary conditions are now

$$
\begin{array}{ll}
\dot{M}_{\mathrm{XY}}=\dot{M}_{\mathrm{disk}} & v_{\mathrm{ff}}=\left[2 G M\left(1 / R-1 / R_{\mathrm{H}}\right)\right]^{1 / 2} \\
P=P_{\mathrm{neb}}+\frac{\dot{M}_{\mathrm{XY}}}{4 \pi R^{2}} v_{\mathrm{ff}}+\frac{2 g}{3 \kappa} & \tau=\max \left(\rho_{\mathrm{neb}} \kappa_{\mathrm{neb}} R, 2 / 3\right) \\
T_{\text {int }}^{4}=\frac{3 \tau L_{\mathrm{int}}}{8 \pi \sigma R^{2}} & T^{4}=(1-A) T_{\mathrm{neb}}^{4}+T_{\mathrm{int}}^{4}
\end{array}
$$

where $A$ is the albedo (assumed to be the same as for Jupiter) and $g=G M / R^{2}$, and for the luminosity we still have $l(R)=L_{\text {int }}$. 


\section{Evolutionary (or isolated) phase}

The last phase starts when the gaseous disk disappears so that the planet evolves at constant mass (we neglect evaporation, as the minimal allowed semimajor axis $a$ is $0.1 \mathrm{AU}$ in the synthesis). During this phase, we use simple standard stellar boundary conditions in the Eddington approximation and write (e.g. Chandrasekhar 1939)

$$
\begin{array}{ll}
P=\frac{2 g}{3 \kappa} & T_{\text {int }}^{4}=\frac{L_{\text {int }}}{4 \pi \sigma R^{2}} \\
T_{\text {equi }}=280 \mathrm{~K}\left(\frac{a}{1 \mathrm{AU}}\right)^{-\frac{1}{2}}\left(\frac{M_{*}}{M_{\odot}}\right) & T^{4}=(1-A) T_{\text {equi }}^{4}+T_{\text {int }}^{4}
\end{array}
$$

and $l(R)=L_{\text {int }}$ which is now also equal the total luminosity $L$. It is clear that the long term evolution is described in a much simpler way than in the Burrows et al. (1997, hereafter BM97) or Baraffe et al. (2003, hereafter BC03) models which employ proper non-gray atmospheres (see Chabrier \& Baraffe 1997). We have however found that the general agreement in terms of total luminosity or radius is good (cf. sections 3. and 4.).

\section{Formation of Jupiter}

The formation of Jupiter is of particular importance as a benchmark for all giant planet formation models. This is because for no other giant planet an equally high number of detailed observational constraints exist. Figure 1 shows the formation of Jupiter in our baseline formation simulation (nJ6).

The initial conditions for this simulation mimic J6 in P96 which in particular means that the initial planetesimal surface density $\Sigma_{\mathrm{p}}$ is $10 \mathrm{~g} / \mathrm{cm}^{3}$, and that the grain opacities are $2 \%$ of the interstellar value.

The simulation shown here is strongly simplified in comparison to the calculations used in the synthesis: in the full model the onset of limited gas accretion (and thus the detachment) as well as the final mass of the planet results in a self-consistent way from the evolution of the gaseous disk (Alibert et al. 2005). Here, the evolution of the disk is switched off. Instead, we fix the maximal allowed $\dot{M}_{\mathrm{XY}}$ to $10^{-3} M_{\oplus} / \mathrm{yr}$ and artificially switch off accretion when the mass of the planet approaches 1 Jupiter mass. Also migration is completely switched off. But in this way we allow for direct comparison with previous works as P96, Hubickyj, Bodenheimer, \& Lissauer (2005) and Lissauer et al. (2009).

The top left and right panel show the characteristic three stages of such in-situ calculations (but all three occur within the attached phase described in sect. 2.3): First a rapid build-up of the core until the isolation mass is reached, then a plateau phase characterized by a slow increase of the envelope mass which allows further core growth, and then the transition to gas runaway accretion. This transition is due to the fact that the Kelvin-Helmholtz time scale $\tau_{\mathrm{KH}}$ decreases with increasing mass.

The cross-over point, i.e. the moment when the core and the envelope mass are identical and equal to $M_{\mathrm{cr}}$ is found to occur at $t_{\mathrm{cr}}=2.14$ Myrs at $M_{\mathrm{cr}}=16.6 M_{\oplus}$. This is in very good agreement with P96 with $t_{\mathrm{cr}}=2.75 \mathrm{Myrs}, M_{\mathrm{cr}}=16.2 M_{\oplus}$ or Hubickyj et al. (2005) who have $t_{\mathrm{cr}}=2.22$ Myrs and $M_{\mathrm{cr}}=16.1 M_{\oplus}$.

Shortly after the cross-over point, the gas accretion rate increase strongly as gas runaway accretion starts, so that it hits the maximal allowed value of $10^{-3} M_{\oplus} / \mathrm{yr}$ at $\mathrm{t}=2.39$ Myrs. The total mass of the planet is at this moment is $65.34 M_{\oplus}$, and $\tau_{\mathrm{KH}}$ is as short as $\sim 2300 \mathrm{yrs}$. This moment is also when the second detached phase begins and the collapse of the envelope starts (cf. Lissauer et al. 2009), which is in 

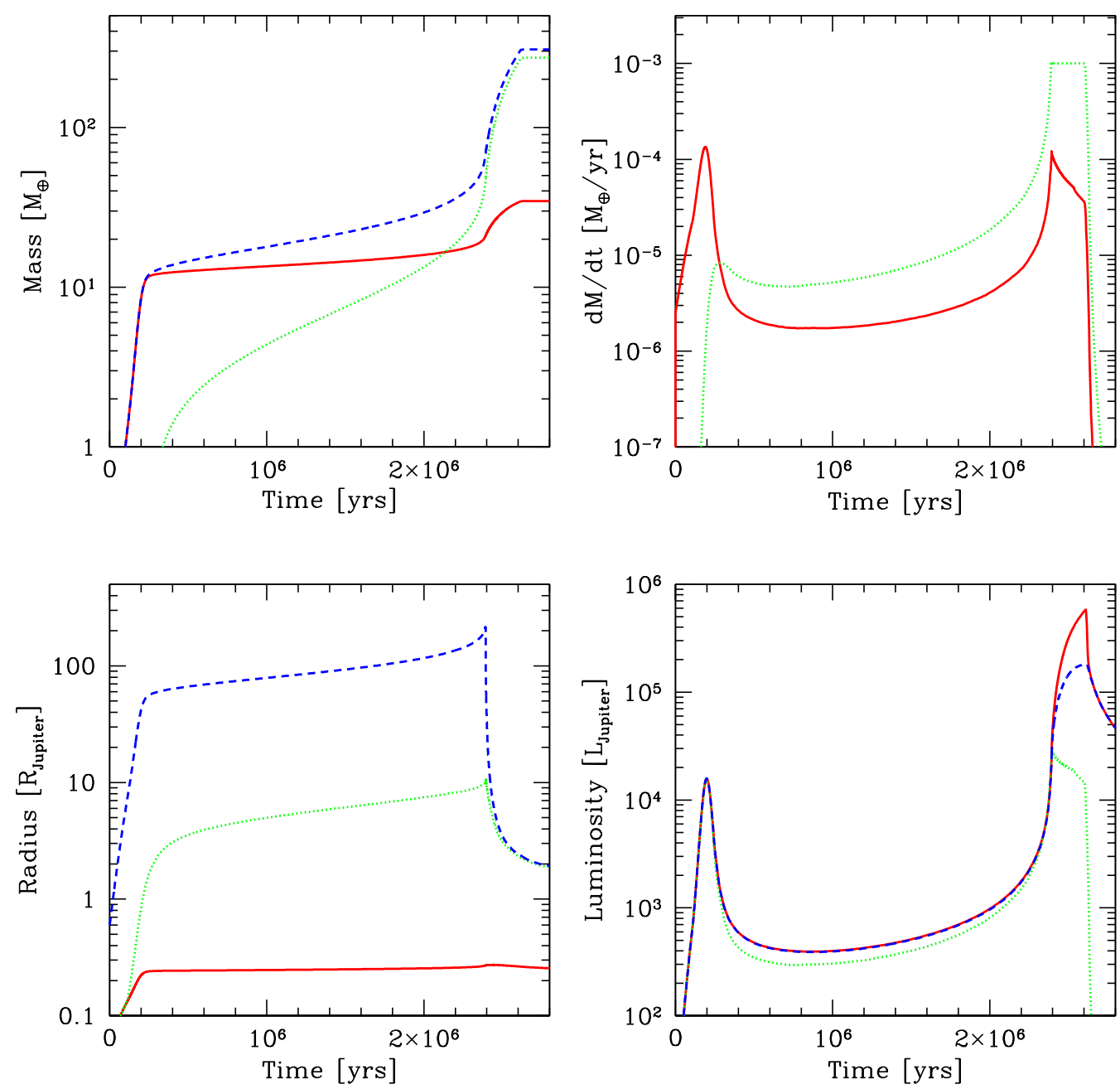

Figure 1.: Simulation nJ6 of the in-situ formation of Jupiter. Top left: evolution of the core mass (red solid line), the envelope mass (green dotted line) and the total mass (blue solid line). Top right: accretion rate of solids $\dot{M}_{\mathrm{Z}}$ (red solid line) and of gas $\dot{M}_{\mathrm{XY}}$ (green dotted line). Bottom left: evolution of the core radius $R_{\text {core }}$ (red solid line), the total radius $R$ (blue dashed line) and the capture radius $R_{\text {capt }}$ (green dotted line). Bottom right: luminosity of the planet in present day intrinsic luminosity of Jupiter. The red solid line is the total luminosity $L$, the blue dashed line is $L_{\text {int }}$ and the green dotted line is $L_{\text {core }}$.

fact a rapid, but still hydrostatic contraction (BP86). About $2 \times 10^{5}$ yrs after this moment, at $\mathrm{t}=2.61$ Myrs, the second luminosity maximum occurs where $L=5.81 \times 10^{5}$ $L_{\mathrm{J}}\left(\log \left(L / L_{\odot}\right)=-3.29\right)$. The maximum occurs just before the gas accretion rate is (artificially) reduced down to zero because the mass of the planet approaches 1 Jupiter mass. The planet has then already contracted to a radius of $2.38 R_{\mathrm{J}}$, and nearly reached its final mass $\left(M=300.6 M_{\oplus}\right)$. The highest temperature at the core-envelope interface 


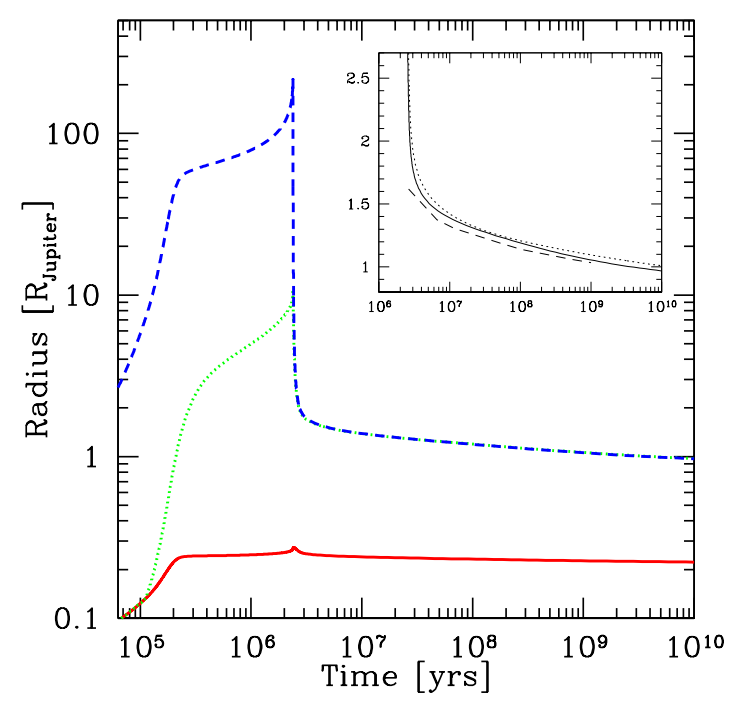

Figure 2.: Radius $R$ (dashed), core radius $R_{\text {core }}$ (solid) and capture radius $R_{\text {capt }}$ (dotted) as a function of time for the nJ6 case. The inset figure is a zoom-in onto the late evolution and shows the radius as found in this work (solid line), in BCO3 (dashed) and BM97 (dotted). Note that the different assumed internal compositions have a certain influence on $R$.

occurs roughly 13000 years after the second luminosity peak, with a $T_{\text {cent }}$ of about $66900 \mathrm{~K}$. Then it starts to fall.

Figure 2 shows the radius of the planet as a function of time, including the long term evolution over Gigayears when the mass is constant. The evolution now occurs slowly by gradual contraction and cooling. For comparison, the temporal evolution of the radius of a $1 M_{\mathrm{J}}$ planet as found by $\mathrm{BC} 03$ and $\mathrm{BM} 97$ is also shown. One finds good agreement.

The planet has the following key properties after 4.6 Gyrs: A total mass of 307.3 $M_{\oplus}$, a heavy element mass $M_{\mathrm{Z}}=34.6 M_{\oplus}(\mathrm{Z}=0.11)$ which falls well inside the (wide) range of possible values for Jupiter of 10 to $42 M_{\oplus}$ (Guillot \& Gautier 2007), a radius of $0.99 R_{\mathrm{J}}$, an intrinsic luminosity of $1.78 L_{\mathrm{J}}$, and a surface temperature $T=133$ $\mathrm{K}$ (the measured value is $124 \mathrm{~K}$, Guillot \& Gautier 2007). The temperature at the core-envelope interface is about $17300 \mathrm{~K}$, similar to what is found in more complex models (Guillot \& Gautier 2007). We thus see that the model, in particular with the simplifications we made, is able to fulfill the most important observational constraints coming from Jupiter.

\section{Luminosity evolution}

Figure 3 shows the luminosity as a function of time for five planetary masses $(10,5,2$, 1 , and $0.5 M_{\mathrm{J}}$ ). The formation phase for all five planets is identical to case nJ6, except that accretion was shut off at these masses.

In the figure, we have also plotted the luminosity in the BM97 and BC03 models. The moment corresponding to $t=0$ of these models has been set to the moment when accretion stops in our simulation. The sharp drop of $L$ particularly well visible for the 10 and $5 M_{\mathrm{J}}$ cases also corresponds to the end of accretion, when $L_{\text {ext }}$ vanishes.

At late times, we see a generally good agreement. One notes that our models might have a certain tendency towards higher luminosities. This could be a consequence of the simple Eddington boundary conditions, in contrast to the atmospheric models used in BM97 and BC03. The discrepancies are however not larger than those mutually between BM97 and BC03. 


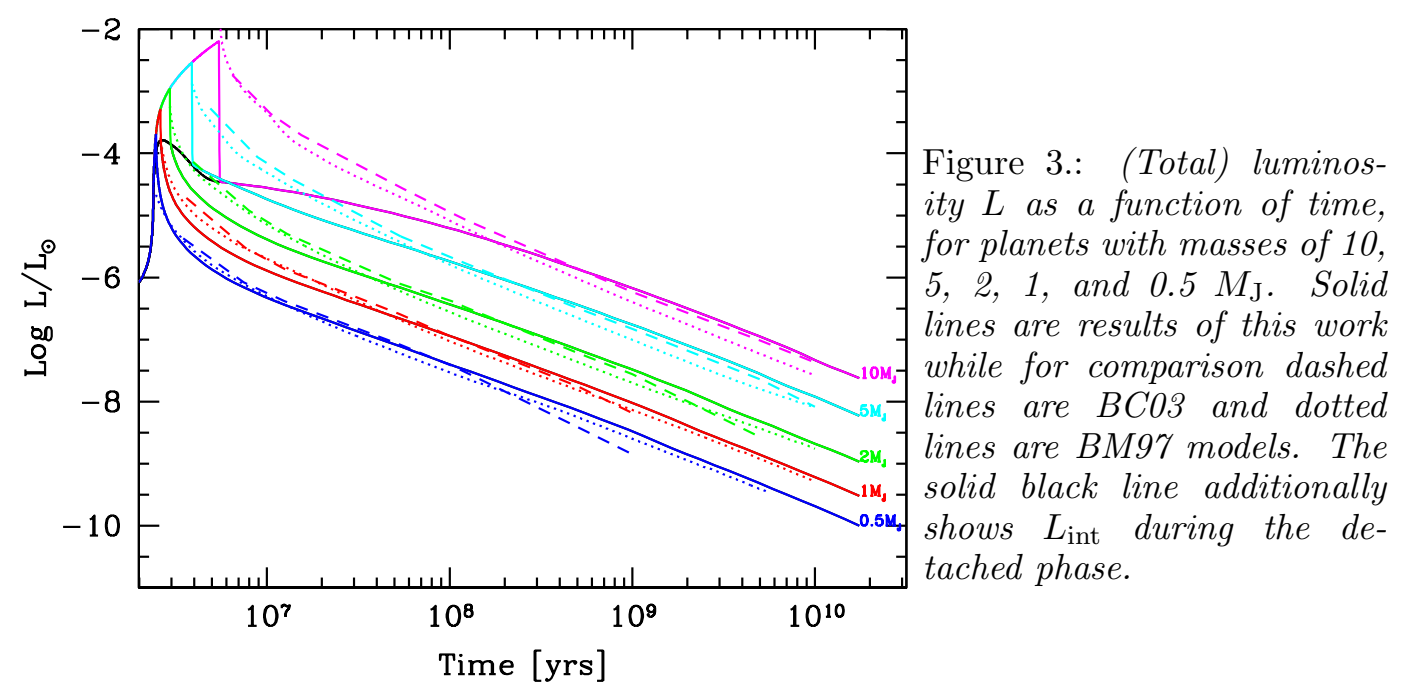

At early times however, systematic discrepancies exist. The luminosities of the planets as found here are smaller than in the other two models. The strength of the difference increases with planetary mass. While no clear difference can be seen for the $0.5 M_{\mathrm{J}}$ case, and only a small one for the $1 M_{\mathrm{J}}$ case, it becomes very marked for the 5 and $10 M_{\mathrm{J}}$ case. Also the duration of the luminosity deficit increases with mass, and becomes as long as a few 100 Myrs for the most massive planet.

This is of course nothing else than the well know difference between "cold start" and "hot start" models (Fortney et al. 2005, Marley et al. 2007) which is thus recovered in our simulations. There are however also differences to Marley et al. (2007): we note for example that in our work, more massive planets are also more luminous than smaller ones (except for a very short phase of the $10 M_{\mathrm{J}}$ planet), in contrast to Marley et al. (2007). It also seems that the luminosity deficit is in general less strong in our simulations. This will be further studied in future work.

\section{Synthetic mass-radius diagram}

With the formation model presented in section 2. it is possible to obtain a synthetic mass-radius diagram by planetary population synthesis (Ida \& Lin 2004, Mordasini, Alibert, \& Benz 2009a).

The probability distributions for the Monte Carlo variables, as well as the synthesis parameters like for example the $\alpha$ parameter for the disk viscosity, or the efficiency factor for type I migration are the same as in Mordasini et al. (2009a). The initial gas surface density profile however is now taken to be $\Sigma(r, t=0)=$ $\Sigma_{0}(r / 5.2 \mathrm{AU})^{-0.9} \exp \left(-(r / 30 \mathrm{AU})^{1.1}\right)$ as indicated by the observation of young circumstellar disks (Andrews et al. 2009). The stellar mass is fixed to $1 M_{\odot}$.

Figure 4 shows the temporal evolution of the synthetic mass-radius diagram in four panels. The color coding indicates the fraction of heavy elements $Z=M_{\mathrm{Z}} / M$. We assume for simplicity in all simulations that all heavy elements reside in the core, so that $M_{\mathrm{Z}}=M_{\text {core }}$.

The top left panels shows the $M-R$ diagram in an early stage of the formation phase at $t=7 \times 10^{5}$ years. The first giant planets have already formed which is possible in metal rich, massive disks. One can distinguish two groups in the diagram: Low mass, 
Detection and Dynamics of Transiting Exoplanets
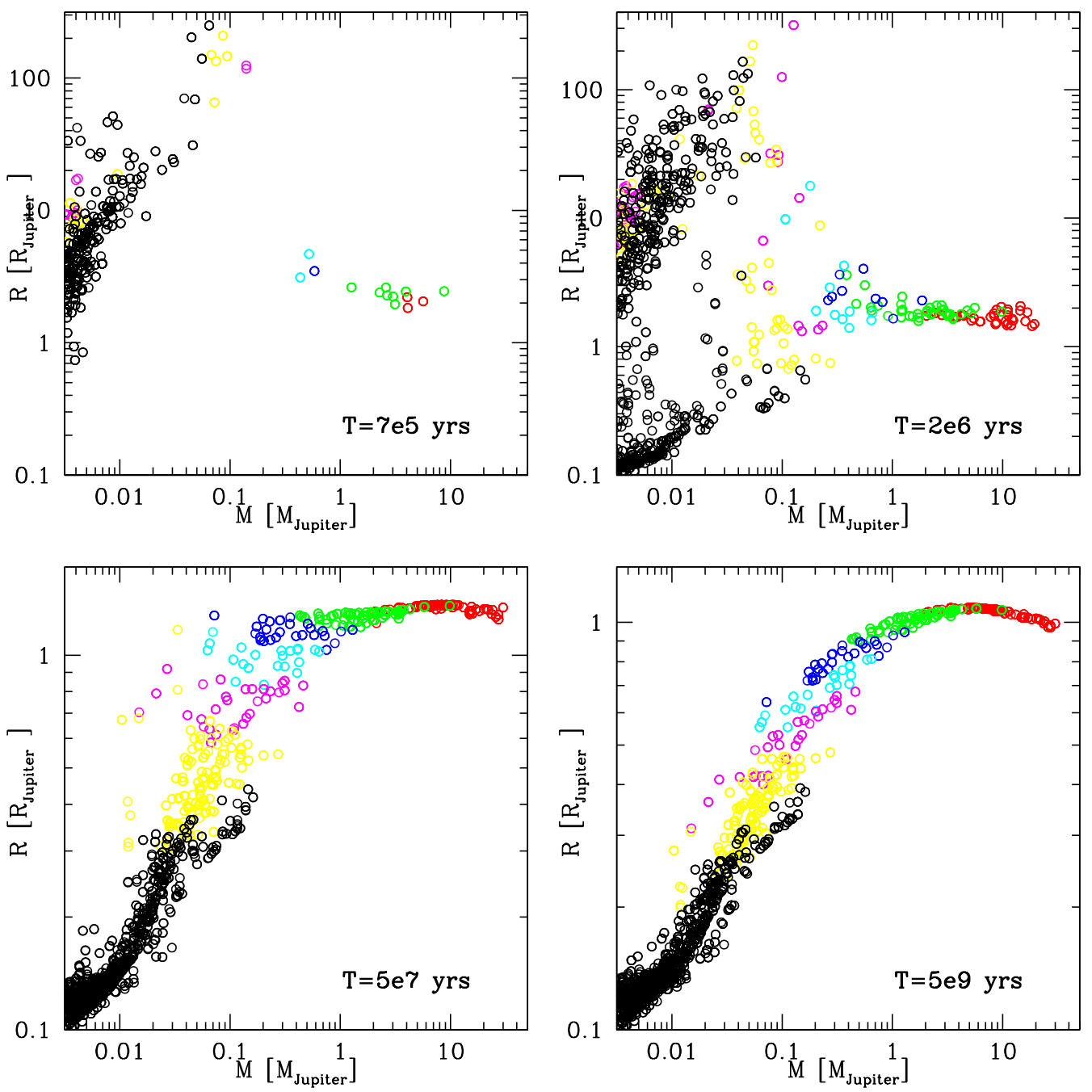

Figure 4.: Temporal evolution of the mass-radius diagram for synthetic planets. The minimal mass corresponds to $1 M_{\oplus}$. Four moments in time are shown, as indicated in the panels. The colors show the fraction of heavy elements in the planet. Red: $Z<5 \%$. Green: $5<Z<20 \%$. Blue: $20<Z<40 \%$. Cyan: $40<Z<60 \%$. Magenta: $60<Z<80 \%$. Yellow: $80<Z<95 \%$. Black: $Z>95 \%$.

pre-collapse (and pre-runaway) planets which have a very large radius $\left(\sim R_{\mathrm{H}}\right)$, and higher mass post-collapse planets which are in runaway gas accretion, with a radius of roughly $2 R_{\mathrm{J}}$. One notes that the transition from one group to the other occurs at a mass of about $0.2 M_{\mathrm{J}} \approx 60 M_{\oplus}$, as we have seen for the Jupiter simulations. The colors show that the core and the envelope have at this point about the same mass, again as seen in the Jupiter models.

The top right panel shows the situation at 2 Myrs. This corresponds to the middle of the formation phase. One sees that there are now also low mass planets with small radii. This are planets which never undergo a phase of runaway gas accretion but collapse after the protoplanetary disk has disappeared. 
The bottom left panel at $t=50$ Myrs shows the early stage of the evolution phase. Mass accretion is now over for about 30 Myrs, and all planets have collapsed, so that the planets only slowly cool and shrink at constant mass. The radius of typical giant planets is now between 1.2 and $1.4 R_{\mathrm{J}}$. There is still a larger spread in $R$ for giant planets of nearly identical masses than later on. This is because the formation history still plays a role.

The bottom right panel finally shows mature planets at an age of $5 \times 10^{9}$ years. For giant planets we see the characteristic maximum of the radius (found here to occur at about $4 M_{\mathrm{J}}$ ) which is due to the change of the degree of degeneracy in the interior (e.g. Chabrier et al. 2009). We also note that at given mass, planets with a higher Z have a smaller radius, as it must be (e.g. Fortney, Marley, \& Barnes 2007).

Concerning the overall shape, we see that low mass planets have small radii, as they consist mostly of solids, while high mass planets have large radii, as they consist mostly of gas, as indicated by the color coding showing the decrease of $Z$ with $M$. This is a consequence of the fact that low mass cores cannot accrete large amounts of gas. In a symmetrically way to this must massive cores always accrete significant amounts of gas (at least if they form during the lifetime of the gaseous disk) as they must trigger the collapse of the envelope. Neither a core-free e.g. $10 M_{\oplus}$ planet (with $R \sim 1 R_{\mathrm{J}}$ at 1 AU, Fortney et al. 2007), nor a pure ice $3 M_{\mathrm{J}}$ planet (with $R \sim 0.4 R_{\mathrm{J}}$, Charbonneau et al. 2009) is formed. We see that the synthetic $M-R$ relationship contains the imprint of the basic concepts of the core accretion formation theory.

\subsection{Comparison with observation}

Figure 5 shows the synthetic $M-R$ diagram at 5 Gyrs (now in linear units for $R$ ), together with the planets of the solar system, and transiting extrasolar planets with $R<1.6 R_{\mathrm{J}}$.

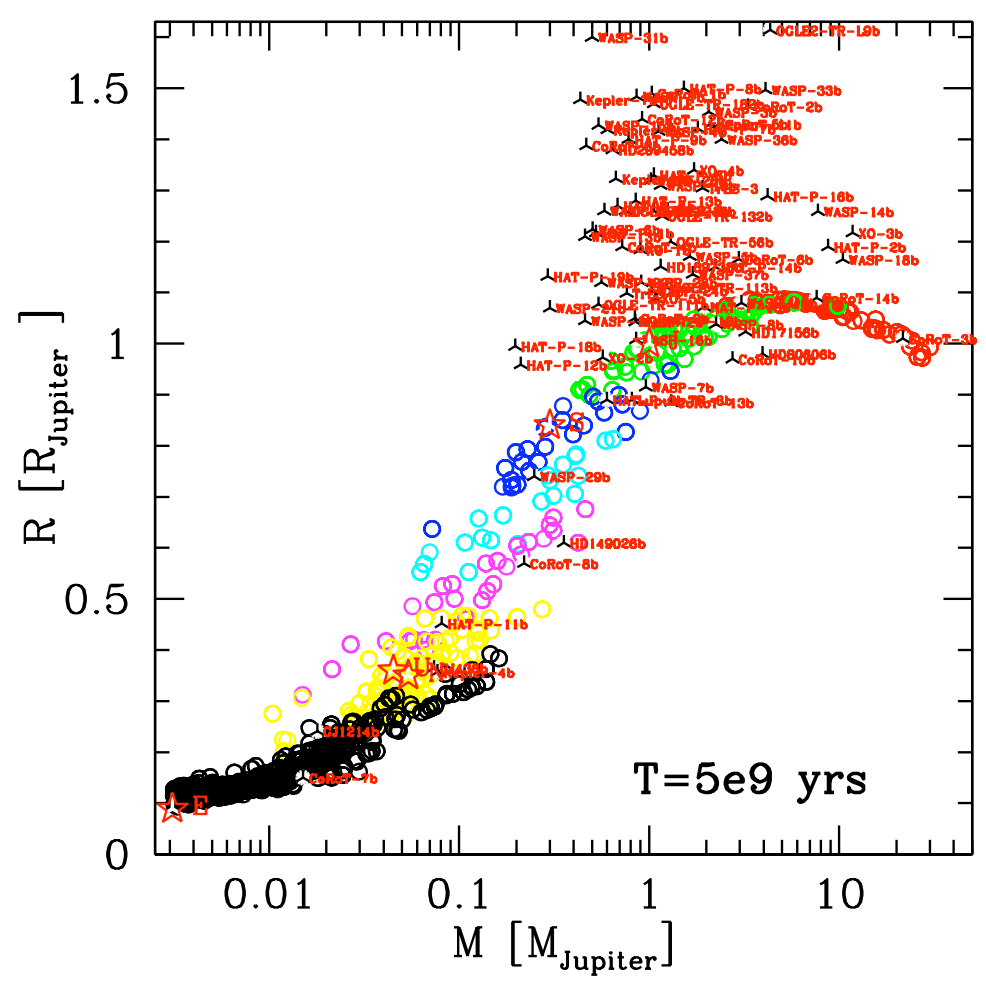

Figure 5.: Mass-radius diagram for synthetic planets at an age of $5 \times 10^{9}$ yrs (same as Fig. 4, bottom right) compared to the planets of the solar system (large symbols labelled $E, J, S$, $U, N)$ and to transiting extrasolar planets. The colors again shows for the synthetic planets the fraction $Z$ of heavy elements, as in Figure. 4. 

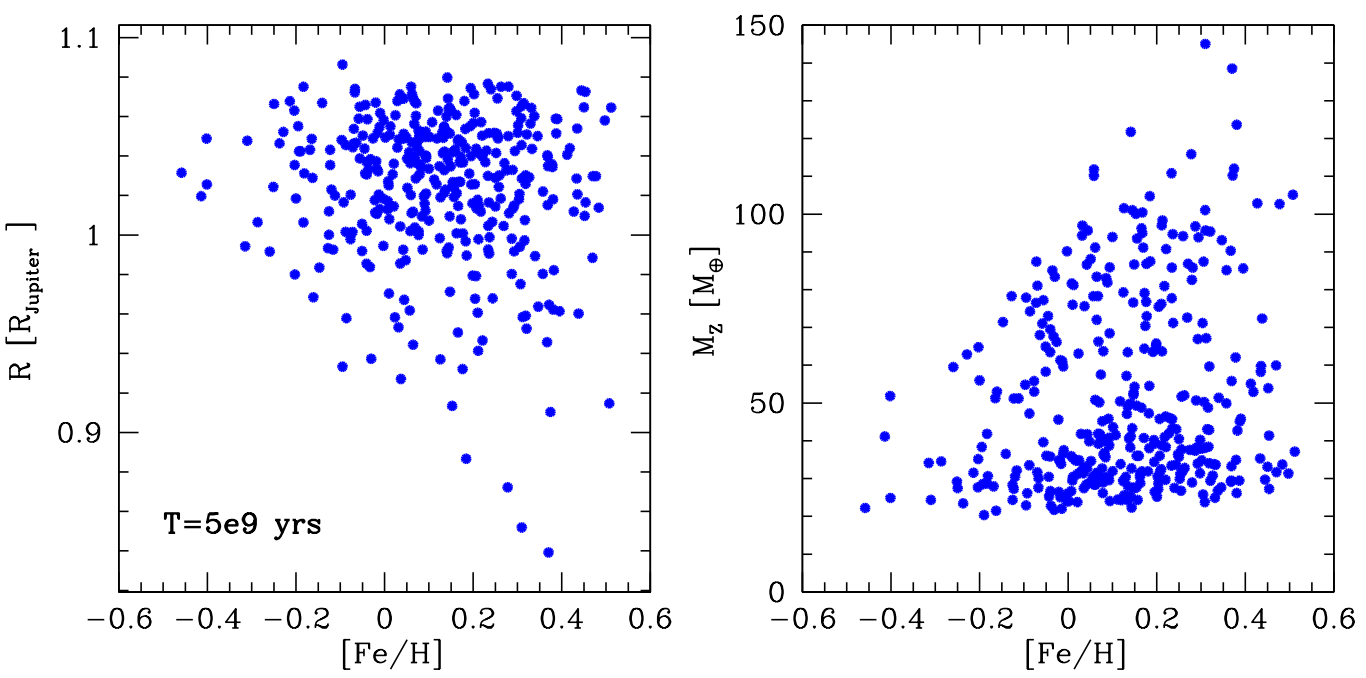

Figure 6.: Left panel: Planetary radius at 5 Gyrs for synthetic planets with a mass of 1 to $3 M_{\mathrm{J}}$ as a function of stellar/disk $[\mathrm{Fe} / \mathrm{H}]$. Right panel: Mass of heavy elements $\mathrm{M}_{\mathrm{Z}}$ inside the same synthetic planets, again as a function of $[\mathrm{Fe} / \mathrm{H}]$.

Regarding the comparison with the planets of the solar system we note a good agreement for the gas giants and the ice giants both in terms of their position in the $R-M$ diagram, as well as in terms of the bulk composition as indicated by the color code.

When making the comparison with the extrasolar planets one should bear in mind that the synthetic planets have all semimajor axes larger than $0.1 \mathrm{AU}$, and that no extra mechanism which could cause inflation is included here. It is therefore no surprise to see that the transiting planets which have radii larger than about $1.1 R_{\mathrm{J}}$ cannot be reproduced.

For transiting exoplanets of a lower mass we see a good agreement between predicted and observed M-R loci, including planets as different as Corot-7b (Léger et al. 2009) or GJ 1214b (Charbonneau et al. 2009). It is interesting to note that intermediate mass planets (with a mass say between Neptune and Saturn), can have for a given mass a large spread of more than a factor 2 for the radius. This reflects the diversity of their heavy element contents, as shown by the colors.

Models of Guillot et al. (2006) and Burrows et al. (2007) suggest that planets with a high amount of heavy elements tend to be found around stars with a high $[\mathrm{Fe} / \mathrm{H}]$, a correlation which was reproduced in the synthetic population of Mordasini et al. (2009b). Figure 6, left panel, shows the radius of synthetic planets at 5 Gyrs with a mass between 1 and $3 M_{\mathrm{J}}$ as a function of the stellar (and thus disk, see Mordasini et al. 2009a) $[\mathrm{Fe} / \mathrm{H}]$. One notes that the minimal radius decreases with increasing stellar metallicity. This is because the maximal core mass increases with $[\mathrm{Fe} / \mathrm{H}]$, as can be seen in the right panel. The maximal radius however hardly changes with metallicity, as all planets contain at least $20 M_{\oplus}$ of heavy elements, which is necessary to trigger gas runaway accretion, and this number nearly does not increase with $[\mathrm{Fe} / \mathrm{H}]$. This is also because at high $[\mathrm{Fe} / \mathrm{H}]$, low absolute amounts of solids can exist in a disk, if the gas mass of the disk is small. On the other hand, there are no planets with a small $R$ 


\section{EPJ Web of Conferences}

at low $[\mathrm{Fe} / \mathrm{H}]$, as metal poor disks do not dispose of the necessary high quantities of metals for incorporation into the planets.

\section{Conclusions}

We have presented an updated planet formation code which allows to calculate in a self-consistent way planetary radii and luminosities during the entire formation and evolution phase. We have then studied with it the formation of Jupiter in the in-situ approximation, and compared the results to other works, finding good agreement during the formation phase. Even though clearly simplified compared to other evolutionary models, we have found reasonable agreement also for the long term evolution. We have for example also found the discrepancy between "hot start" and "cold start" models.

Finally, we have calculated a synthetic planetary population around solar-like stars and presented in this way how the planetary mass-radius diagram comes into existence. We show that the general shape of the observed and the synthetic mass-radius relationship is in good agreement. The exception are bloated Hot Jupiters which can only be explained with additional mechanism which are related to their proximity to the host star. The general shape of the $M-R$ relation can be understood by a decrease of the heavy element fraction with mass. This is a direct consequence of the core accretion theory: low mass planets cannot accrete massive gas envelopes because of their long cooling timescale. Massive cores $\left(\sim 20 M_{\oplus}\right)$ on the other hand trigger gas runaway accretion. The spread of planetary radii for one given mass can however by substantial due to different formation histories. We thus conclude that the observed $M-R$ diagram carries the imprint of the planetary formation mechanism.

Acknowledgements. We thank K.-M. Dittkrist, C. Ormel, C. Broeg, J. Leconte, I. Baraffe and G. Chabrier for useful discussions. C. M. acknowledges the support as a fellow of the Alexander von Humboldt foundation, and the hospitality of the Kavli Institute for Theoretical Physics at UCSB in spring 2010 (funded by the U. S. NSF through Grant PHY05-51164). Y. A. is thankful for the support by the European Research Council under grant 239605.

\section{References}

Alibert, Y., Mordasini, C., \& Benz, W. 2004, A\&A, 417, L25

Alibert, Y., Mordasini, C., Benz, W., \& Winisdoerffer, C. 2005, A\&A, 434, 343

Andrews, S. M., Wilner, D. J., Hughes, A. M., Qi, C., \& Dullemond, C. P. 2009, ApJ, 700, 1502

Baraffe, I., Chabrier, G., Barman, T. S., Allard, F., \& Hauschildt, P. H. 2003, A\&A, 402, 701

Baraffe, I., Chabrier, G., \& Barman, T. 2008, A\&A, 482, 315

Broeg, C. H. 2009, Icarus, 204, 15

Bodenheimer, P., \& Pollack, J. B. 1986, Icarus, 67, 391

Bodenheimer, P., Hubickyj, O., \& Lissauer, J. J. 2000, Icarus, 143, 2 2010, arXiv:1006.2949

Burrows, A., Marley, M., Hubbard, W. B., Lunine, J. I., Guillot, T., Saumon, D., et al. 1997, ApJ, 491, 856

Burrows, A., Hubeny, I., Budaj, J., \& Hubbard, W. B. 2007, ApJ, 661, 502

Chabrier, G., \& Baraffe, I. 1997, A\&A, 327, 1039

Chabrier, G., Baraffe, I., Leconte, J., Gallardo, J., \& Barman, T. 2009, American Institute of Physics Conference Series, 1094, 102

Chandrasekhar S. 1939. Stellar Structure and Evolution. Univ. Chicago Press, Chicago 


\section{Detection and Dynamics of Transiting Exoplanets}

Charbonneau, D., Berta, Z. K., Irwin, J., Burke, C. J., Nutzman, P., Buchhave, L. A., et al. 2009, Nature , 462, 891

Figueira, P., Pont, F., Mordasini, C., Alibert, Y., Georgy, C., \& Benz, W. 2009, A\&A, 493, 671

Fortney, J. J., Marley, M. S., Hubickyj, O., Bodenheimer, P., \& Lissauer, J. J. 2005, Astronomische Nachrichten, 326, 925

Fortney, J. J., Marley, M. S., \& Barnes, J. W. 2007, ApJ, 659, 1661

Greenzweig, Y., \& Lissauer, J. J. 1992, Icarus, 87, 40

Guillot, T. 2005, Annu. Rev. Earth Planet. Sci., 33, 493

Guillot, T., Santos, N. C., Pont, F., Iro, N, Melo, C., \& Ribas, I. 2006, A\&A, 453, L21

Guillot, T., \& Gautier, D. 2007, in Treatise of Geophysics, vol. 10, Planets and Moons, ed. Schubert G., \&, Spohn T., 439

Hubickyj, O., Bodenheimer, P., \& Lissauer, J. J. 2005, Icarus, 179, 415

Ida, S., \& Lin, D. N. C. 2004, ApJ, 604, 388

Leconte, J., Baraffe, I., Chabrier, G., Barman, T., \& Levrard, B. 2009, A\&A, 506, 385

Léger, A., Rouan, D., Schneider, J., Barge, P., Fridlund, M., Samuel, B., et al. 2009, A\&A, 506, 287

Lissauer, J. J. 1993, ARA\&A, 31, 129

Lissauer, J. J., Hubickyj, O., D’Angelo, G., \& Bodenheimer, P. 2009, Icarus, 199, 338

Lubow, S. H., Seibert, M., \& Artymowicz, P. 1999, ApJ, 526, 1001

Marley, M. S., Fortney, J. J., Hubickyj, O., Bodenheimer, P., \& Lissauer, J. J. 2007, ApJ, 655, 541

Mizuno H., Nakazawa K., \& Hayashi C. 1978, Progress of Theoretical Physics 60:699

Mordasini, C., Alibert, Y., \& Benz, W. 2009a, A\&A, 501, 1139

Mordasini, C., Alibert, Y., Benz, W., \& Naef, D. 2009b, A\&A, 501, 1161

Papaloizou, J. C. B., \& Terquem, C. 1999, ApJ, 521, 823

Papaloizou, J. C. B., \& Nelson, R. P. 2005, A\&A, 433, 247

Perri, F., \& Cameron, A. G. W. 1974, Icarus, 22, 416

Pollack, J. B., Hubickyj, O., Bodenheimer, P., Lissauer, J. J., Podolak, M., \& Greenzweig, Y. 1996, Icarus, 124, 62

Safronov V. S 1969, Evolution of the Protoplanetary Cloud and Formation of the Earth and the Planets. Nauka, Moscow

Stahler, S. W., Shu, F. H., \& Taam, R. E. 1980, ApJ, 241, 637 